\title{
APPLICATION AND USE OF ULTRASONICS
}

GOUND waves are capable of travelling through any $\checkmark$ elastic medium, whether solid, liquid or gas. Within the frequency-range of approximately 16$16,000 \mathrm{c} . / \mathrm{s}$. the waves are detectable by the human ear, but at greater than $16,000 \mathrm{c} . / \mathrm{s}$. they are inaudible and are termed ultrasonic.

The use of ultrasonic energy in industrial processes has grown rapidly in recent years, and in the illustrated booklet, Using Ultrasonics*, issued by the Department of Scientific and Industrial Research, a wide range of present-day applications, including non-destructive testing, cleaning and machining, are reviewed. The author is $\mathrm{R}$. Hamilton, the industrial correspondent of Electronics Weekly.

In modern forms of generators of ultrasonic energy, the energy at the required frequency is first generated by electronic oscillators and then converted into mechanical energy by one of two basic methods utilizing transducers. The transducer sets up periodic vibrations at the ultrasonic frequency and when placed in contact with the medium causes ultrasonic energy to be transmitted into the medium, usually in the form of longitudinal waves. The piezo-electric transducer uses the piezo-electric effect of crystals, such as quartz, Rochelle salt,

* Using Ultrasonics. By R. Hamilton. Pp. 16. (London: Department of Scientific and Industrial Research. 1961). barium titanate, or lead-zirconate-titanate, and the magneto-strictive transducer utilizes the change in dimensions of a ferro-magnetic material when a magnetic field is applied. Ultrasonic applications consist of two categories-low-power systems, including echo sounding, burglar alarms and flaw detection, and high-power processes for cleaning, machining and welding.

Various flow-detection techniques are discussed in the booklet, together with other non-destructive tests of materials using ultrasonics. The use of ultrasonics in geology, in delay lines for electronic computers or in radar installations, and for the measurement of the electric constants of solids, are also briefly described. The greatest hindrance to efficient soldering is the tendency of the metals to be soldered to form oxides when heat is applied. Using ultrasonic methods satisfactory joints can be made, even with aluminium, without using a flux. Both grinding and drilling can be performed by ultrasonic methods, the tool acting not directly but through an abrasive slurry. Holes can be cut to any required shape, and the hard materials which can be cut satisfactorily include glass, quartz, ferrites, ceramics, tungsten carbide and uranium. Wafers of germanium and silicon for use in transistors can also be sliced by this method.

\section{ELECTRON MICROSCOPE STUDIES OF COCCOLITHS IN OCEANIC DEPOSITS}

$\mathrm{T}$ O-DAY the coccolithophorids form one of the three main groups of autotrophic plankton algae in the sea. The biogeographical and quantitative studies of this group which mainly have been carried out in the Atlantic Ocean and the Mediterranean indicate that they play an essential part in oceanic primary production.

The calcareous coccoliths which cover the coccolithophorid cells offer the main basis for the taxonomic division, although other morphological characters are also used. When ten years ago electron microscopy was applied in the study of coccolith morphology ${ }^{1}$ it became evident that the light microscope gives only incomplete information on the structure of the coccoliths and that electron microscopy is essential for an adequate description of these plankton algae.

In marine sediments and fossil deposits the coccoliths are the only remains of the coccolithophorid cells which are preserved and even these may have been fragmented or partly damaged. By means of electron microscopy it is, however, possible to study the morphology of the coccoliths in such material, and, since the microstructure is characteristic of each species, their identity may be ascertained, even in the case of very small specimens.

A recent communication by Maurice Black and Barbara Barnes ${ }^{2}$ affords an excellent illustration of what may be obtained by the use of electron microscopy of carbon replicas of coccoliths from sediments. This first report deals with observations on material from a depth of $1,990 \mathrm{f}$. at a Challenger station in the South Atlantic Ocean and gives descriptions of ten of the more than fifty species present in samples which the authors are studying ranging in age from presentday ooze to early Tertiary material. Three species are described as new, the remainder being referred to species previously observed in plankton samples or fossil material. Instructive micrographs are presented for most of the species.

The study of oceanic cores where sediments from a very long range of time are present, as carried out by Black and Barnes, will serve to connect the observations on recent populations with those on material from previous periods.

The coccolithophorids now occurring in the oceans are still inadequately studied. The older descriptions are gradually being revised on the basis of electronmicroscopical observations and many new species have been described ${ }^{3-5}$. Some of these have been found to have characteristic distribution patterns. Cultures have proved to be useful for obtaining information on their life-history, but essential details in their morphology, such as flagellation, remain unknown even in common species. Experimental work on their physiology is only in its prime. Further investigations of material from water samples, sediments and fossil deposits are necessary in order to obtain material for a satisfactory taxonomic treatment of the group, but the documentation of a lifecycle in Coccolithus pelagicus, which includes stages with two different types of coccoliths ${ }^{6}$, demonstrates 